\title{
A Generalization of Distributed Consensus Algorithms for Wireless Sensor Networks
}

\author{
Jiinho Choi \\ Gwangju Institute of Science and Technology (GIST), Korea
}

\begin{abstract}
In wireless sensor networks, distributed consensus algorithms can be employed for distributed detection. Each sensor node can compute its log-likelihood ratio (LLR) from localobservations for a target event and using an iterative distributed algorithm, the average of sensors' LLRs can be available to all the sensor nodes. While the average of sensors' LLRs allows eachsensor node to make a final decision as a decision statistic for an overall detection problem with all sensors' LLRs, it may be desirable if all sensors' LLRs or local observations, which form a full information vector and denoted by $x$, could be available to each sensor for other purposes more than the detection of a target event. In this paper, we show that each sensor can have not only the average of local observations, but also full information vector, $x$, (or its estimate) using a wellknown iterative distributed algorithm.
\end{abstract}

Keywords: Internet of Things; Consensus algorithms; Wireless Sensor Networks

\section{Introduction}

Wireless sensor networks (WSNs) have various applications including environmental monitoring and surveillance [1], [2] and become part of the Internet of Things (IoT) [3]. A WSN consists of a number of sensor nodes that have wireless transceivers to communicate with other adjacent sensor nodes or a central unit. In the context of distributed detection [4], this central unit is called a fusion center (FC) as all sensors' local decisions regarding a target event are collected and combined (or fused) for a final decision. In the presence of a FC, each sensor node only makes a local decision. For example, in distributed detection, each sensor node can make a local decision and transmit it to the FC [5], [6], [7], [8]. The detection performance at the FC depends on the number of sensors if local decisions at sensors can be correctly received at the FC. However, if local decisions from sensors are not reliably received at the FC due to wireless channel impairments (e.g., fading and background noise), the channel conditions also affect on the performance [9], [10], [11], [12]. In general, the signals from sensor nodes that are far away from the FC may not be reliably received at the FC unless they use high transmission powers, which is not desirable as sensor nodes could have limited power sources (e.g., batteries). In the centralized approach with a FC, when the final decision is required at sensor nodes for further processing, the FC can broadcast it to sensors. However, in this case, the sensors that are far away may not reliably receive this final decision.

Distributed consensus algorithms [13], [14], [15], [16] can be employed for WSNs to overcome the problems pointed out above. As distributed consensus algorithms only require local communications between neighbor sensor nodes, the transmission power could be lower than that in the centralized approach with a FC for distributed detection. Through iterative information exchanges between neighbor sensor nodes, the consensus or averaging can be achieved at all the sensor nodes. As shown in [15], [16], each sensor can have the average of all sensors' log-likelihood ratios (LLRs) by an iterative distributed algorithm for distributed detection. In [17], distributed synchronization can also be carried out using consensus algorithms. As each signal transmission for 
information exchange requires a certain amount of energy consumption, in general, iterative distributed algorithms of fast convergence rate are required [14], [18]. Furthermore, as the signals for information exchange are to be quantized for digital wireless communications, the impact of quantization on distributed consensus algorithms needs to be investigated [19].

In this paper, we show that a well-known iterative distributed algorithm can be used to provide every sensors' observations to all sensor nodes without any modification. As a result, each sensor node can have the average of sensors' observations as well as an estimate of all sensors' observations.

\section{Main Results}

Distributed consensus algorithms are iterative algorithms that are based on information exchange between neighbor sensor nodes at each iteration. Let $x_{l}(\mathrm{t})$ denote the state variable at sensor $l$ at time $t$ with $x_{l}(\mathrm{t})=x_{l}$, where $t$ is the index for iteration. Then, each sensor can have the average of $x_{l}$,'s by using the following iterative distributed algorithm [13]:

$$
x_{l}(t+1)=x_{l}(t)+\mu \sum_{m \in N_{l}}\left(x_{m}(t)-x_{l}(t)\right), l=1, \ldots, L,
$$

The network topology can be represented by a Laplacian matrix. We have the following results.

Theorem 1. For a graph whose Laplacian matrix has Ld distinct eigenvalues, only Ld - 1 iterations of the iterative distributed algorithm in (1) are required to obtain the mean of $\mathbf{x}$ at every sensor nodes..

Different approaches to find the minimum number of iteration for achiving consensus can also be found in [18]. We have the following consequence of Theorem 2 on the diameter 1 of a graph.

Theorem 2. For a connected and undirected graph $\mathrm{G}$, the diameter, denoted by $\operatorname{diam}(\mathrm{G})$, is upper-bounded as

$$
\operatorname{diam}(G) \leq L_{d}-1
$$

\section{Conclusions}

In this paper, we studied the iterative distributed algorithm to provide an estimate of the full information vector at each sensor node. We have found that this condition is related to a controllability matrix and the order of a partial information vector, as an estimate of $\mathbf{x}$, depends on the number of distinct eigenvalues of a graph. From the latter result, we had two immediate results: i) the minimum number of iterations of the iterative distributed algorithm for achieving consensus is Ld -1 1, where Ld is the number of distinct eigenvalues; ii) the diameter of a connected graph is upper-boundedby Ld - 1 .

\section{Acknowledgements}

This work was supported by GIST Research Institute(GRI) grant funded by GIST in 2018.

\section{References}

[1] A. Swami, Q. Zhao, Y.-W. Hong, and L. Tong, Wireless Sensor Networks, John Wiley \& Sons, 2007. https://doi.org/10.1002/9780470061794

[2] Q. Zhao, A. Swami, and L. Tong, "The interplay between signal processing and networking in sensor networks," IEEE Signal Proc. Mag., vol. 23, no. 4, pp. 84-93, July 2006.

https://doi.org/10.1109/MSP.2006.1657819

[3] I. Khan, F. Belqasmi, R. Glitho, N. Crespi, M. Morrow and P. Polakos, "Wireless sensor network virtualization: A survey,” IEEE Communications Surveys \& Tutorials, vol. 18, no. 1, pp. 553-576, First-quarter 2016.

[4] P. K. Varshney, Distributed Detection and Data Fusion, New York: Springer-Verlag, 1997. 
https://doi.org/10.1007/978-1-4612-1904-0

[5] J. N. Tsitsiklis, "Decenterlized detection by a large number of sensors," Math. of Control, Signals, and Systems, vol. 1, pp. 167-182, 1988. https://doi.org/10.1007/BF02551407

[6] Z. Chair and P. K. Varshney, "Optimal data fusion in multiple sensor detection systems," IEEE Trans. Aerospace and Electronic Sys., vol. 22, no. 1, pp. 98-101, January 1986.

https://doi.org/10.1109/TAES.1986.310699

[7] Q. Zhang, P. K. Varshney, and R. D. Wesel, "Optimal bi-level quantization of i.i.d. sensor observations for binary hypothesis testing,” IEEE Trans. Inform. Theory, vol. 48(7), pp. 2105-2111, July 2002. https://doi.org/10.1109/TIT.2002.1013153

[8] J.-F. Chamberland and V. V. Veeravalli, "Decenterlized detection in sensor networks," IEEE Trans. Signal Proc., vol. 51(2), pp. 407-416, February 2003. https://doi.org/10.1109/TSP.2002.806982

[9] B. Chen, R. Jiang, T. Kasetkasem, and P. K. Varshney, "Channel aware decision fusion in wireless sensor networks," IEEE Trans. Signal Proc., vol. 52, no. 12, pp. 3454-3458, December 2004. https://doi.org/10.1109/TSP.2004.837404

[10]B. Chen, L. Tong, and P. K. Varshney, "Channel-aware distributed detection in wireless sensor networks," IEEE Signal Proc. Mag., vol. 23, no. 4, pp. 16-26, July 2006. https://doi.org/10.1109/MSP.2006.1657814

[11]Q. Cheng, B. Chen, and P. K. Varshney, "Detection performance limits for distributed sensor networks in the presence of nonideal channels," IEEE Trans. Wireless Comm., vol. 5, no. 11, pp. 3034-3038, November 2006. https://doi.org/10.1109/TWC.2006.05147

[12]R. Niu, B. Chen, and P. K. Varshney, "Fusion of decisions transmitted over Rayleigh fading channels in wireless sensor networks,” IEEE Trans. Signal Proc., vol. 54(3), pp. 1018-1027, March 2006. https://doi.org/10.1109/TSP.2005.863033

[13]R. O. Saber and R. M. Murray, "Consensus protocols for networks of dynamic agents," in Proc. IEEE American Control Conf., 2003, pp. 951-956. https://doi.org/10.1109/ACC.2003.1239709

[14] L. Xiao and S. Boyd, "Fast linear iterations for distributed averaging," Systems and Control Letters, vol. 53, pp. 65-78, 2004. https://doi.org/10.1016/j.sysconle.2004.02.022

[15] S. A. Aldosari and J. M. F. Moura, "Distributed detection in sensor networks: connectivity graph and small world networks," in Proc. IEEE 39'th Asilomar Conf. on Signals, Systems, and Computers, 2005, pp. 230-234.

[16]R. Olfati-Saber, E. Franco, E. Frazzoli, and J. S. Shamma, "Belief consensus and distributed hypothesis testing in sensor networks," Lecture Notes in Control and Information Sciences (LNCIS), Networked Embedded Sensing and Control, vo. 331, pp. 169-182, 2006. https://doi.org/10.1007/11533382_11

[17]Y. Jin, X. Zhang and B. Yao, "Distributed synchronization in large-scale wireless sensor networks using group consensus protocol,” International Journal of Distributed Sensor Networks Vol. 13(11), 2017. https://doi.org/10.1177/1550147717718114

[18]R. L. G. Cavalcante and B. Mulgrew, "Adaptive filter algorithms for accelerated discrete-time consensus," IEEE Trans. Signal Proc., vol. 58(3), pp. 1049-1058, March 2010.

https://doi.org/10.1109/TSP.2009.2032450

[19] J. Fang and H. Li, "Distributed consensus with quantized data via sequence averaging," IEEE Trans. Signal Proc., vol. 58(2), pp. 944-948, February 2010. https://doi.org/10.1109/TSP.2009.2032951 